\title{
Comparison of nanoparticle diameter measurements by Atomic Force Microscopy and Scanning Electron Microscopy
}

\author{
A. Delvallée ${ }^{\mathrm{a}}$, N. Feltin, S. Ducourtieux, M. Trabelsi and J-.F Hochepied ${ }^{\mathrm{b}}$ \\ ${ }^{a}$ LNE, DMSI, Pôle de Recherche de Métrologie avancée - 29, avenue Roger Hennequin, 79197 Trappes Cedex, France. \\ ${ }^{\mathrm{b}}$ Mines ParisTech, centre des matériaux, Z.I. Les Glaizes - 5, rue Léon Blum 91120 Palaiseau, France.
}

\begin{abstract}
Using combined AFM (Atomic Force Microscope) and a SEM (Scanning Electron Microscope) makes it possible to accurately measure a nano-object in 3 dimensions. This paper deals with the traceable measurements of the size and the size distribution of a $\mathrm{SiO}_{2} \mathrm{spherical}$ shaped nanoparticle population performed by both microscopy techniques. The complementary nature of AFM and SEM is investigated. The construction of size distribution histogram was carried out by means of a homemade program described here. This semi-automatic program is capable of counting only isolated nanoparticles and eliminating measurement artefacts and aggregates. Finally, a comparison between both measurement types is proposed.
\end{abstract}

\section{CARMEN: the LNE's platform dedicated to the nanometrology}

LNE is developing a platform dedicated to the metrological characterization of nanomaterials (CARMEN platform) that will supply a complete offer for measuring main parameters characterizing a nanoobject: size, shape, size distribution, chemical composition, agglomeration/aggregation state, crystal structure, surface charge, specific surface area. In a first step, the LNE's nanometrology team is focusing on the measurement of the mean diameter and size distribution of a spherical nanoparticles (NPs) population by Atomic Force Microscopy (AFM). From sampling method to statistical tools, through instrument calibration and imaging, we propose a method for traceable measurements of the nanoparticle height. The same approach is developed for Scanning Electron Microscopy (SEM). Thanks to SEM, the lateral dimension of particles can be obtained with a high resolution. In this paper, we investigate the complementary nature of both instruments, AFM and SEM, for a nanoparticle size measurement in the 3 dimensions. Using both techniques induces some constraints on sampling described in this paper.

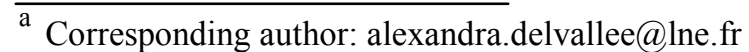

\subsection{Traceability of the measurements at the nanoscale}

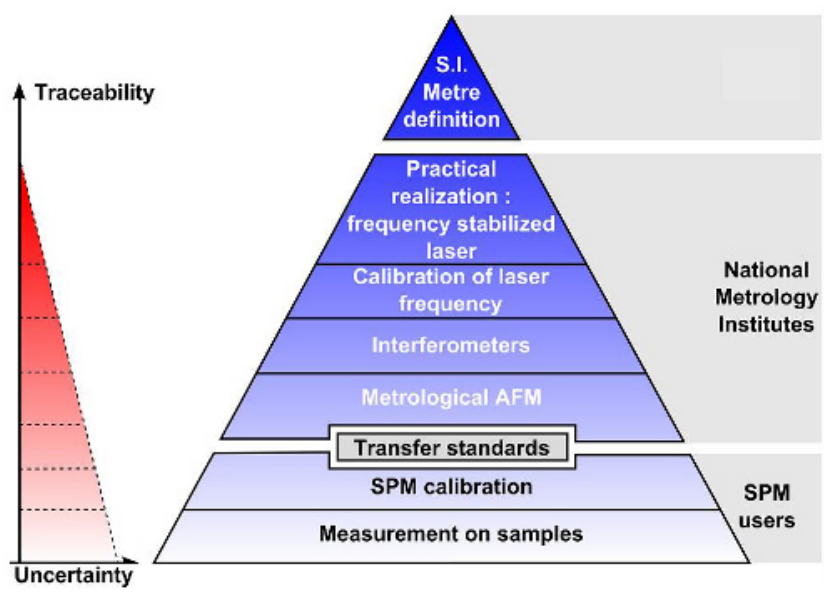

Figure 1 : Traceability routes of a nano-object measurement.

The traceability route of the dimensional measurements at the nanoscale implementing a metrological AFM (mAFM) is reminded in Figure 1. The mAFM is a reference instrument enabling to link the nanoscale dimensional measurements to the SI units through a calibration process performed by means of transfer standards like periodic 1D or 2D gratings. Since 2007, LNE has been developing such an instrument implementing interferometers with laser sources calibrated in wavelength and measuring in real time the relative position of the tip with respect to the surface. Thus, for each point of the image, the XYZ coordinates are assumed to be absolute and, so, are linked to the SI 
units. In practice, this latter instrument is not suitable for daily measurements because the measuring time is too long and the implementation is quite complex. The NP characterization is then carried out through commercial AFM and SEM, which are periodically calibrated by means of standards measured by the mAFM. Consequently, this step ensures the traceability of routine measurements to the SI meter.

\subsection{LNE's AFM and SEM and their environment}

LNE's AFM is a Dimension V (Veeco) equipped with a hybrid three-axis scanner operating under closed loop control which provides a scan range of $90 \mu \mathrm{m} \times 90 \mu \mathrm{m} \times 8$ $\mu \mathrm{m}$. To avoid vibrations during measurements, this AFM is put on an antivibration table and protected with an acoustic enclosure.

The LNE's CARMEN platform is also equipped with a Zeiss UltraPlus FEG (Field Emission Gun) SEM, which can reach $1 \mathrm{~nm}$ lateral resolution at $15 \mathrm{kV}$.

Moreover, those two instruments are placed on massive concrete blocks dissociated from the rest of the building. The laboratory is humidity $(50 \%+/-5 \%)$ and temperature $\left(20^{\circ} \mathrm{C}+/-0.05^{\circ} \mathrm{C}\right)$ stabilized. Thanks to those precautions, the measurement systems are highly stable and measurements are more repeatable.

\section{AFM measurements}

\subsection{AFM measurement principle}

Binnig and Quate invented atomic Force Microscope (AFM) in 1986 [1]. This technique is capable of providing images, almost at the level of atomic resolution, by measuring the topography of the sample. The image is generated by quantifying the forces between the probe and the sample surface.

In the general case, an AFM described in the Figure, consists of a tip located at the end of a cantilever and a feedback control loop.

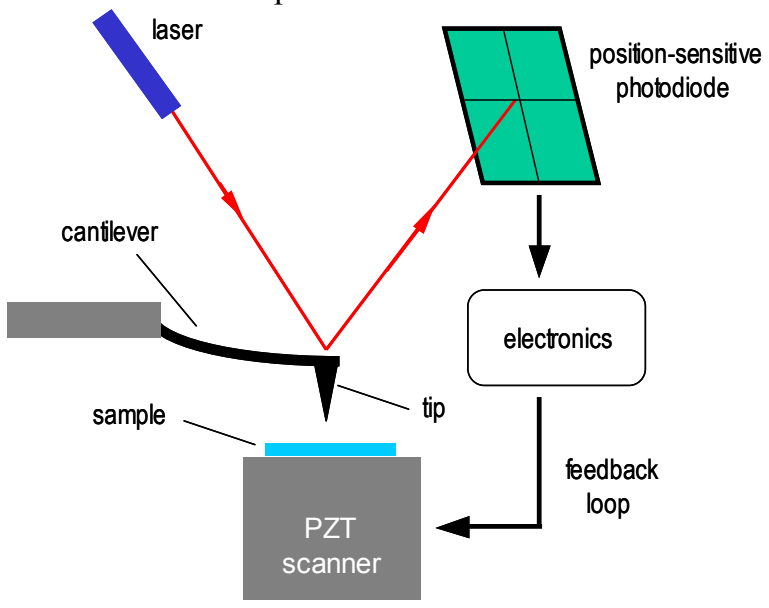

Figure 2: AFM operating principle

AFM technique makes it possible to reach a subnanometer resolution along the vertical axis and the relation between interaction forces $F$ and the tip vertical displacement $\Delta \mathrm{z}$ is written as follows:

$$
F=-K . \Delta \mathrm{z}
$$

Where $K$ denotes the spring constant of the cantilever.

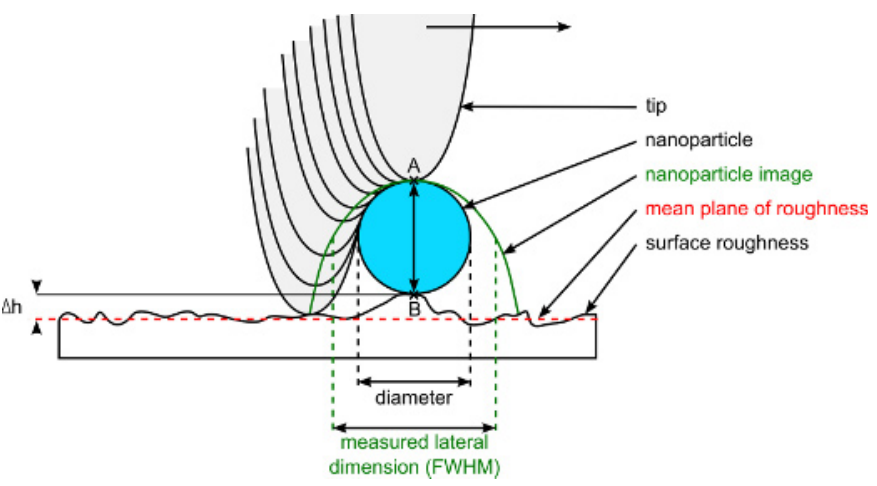

Figure 3 : AFM nanoparticle measurement method and tip effect

On the other hand, as described in Figure 3, the tip size and its geometry lead to a broadening of the lateral dimensions of the nanoparticle imaged by AFM. As a consequence, their real diameter is very difficult to be accurately measured in the surface plane. But, in the case of NP assumed to be spherical, a reliable method for determining the size (diameter) of NPs is to measure the height of nano-object. But for that purpose, certain precautions must be taken in order to avoid the measurement error and minimize the uncertainties. That is the reason why the nanoparticles have to be correctly dispersed and immobilized on a flat substrate. Indeed a minimal distance is required (mean NP size + tip size) between NP to obtain a complete image of each of them. Two points are then needed to precisely measure the height of a nano-object. The first data point corresponds to local maximum on the nanoparticle, the second one is the mean height of the surface topography (figure 3 ). The height of the particle is calculated as the difference of both data. The mean height of the surface topography is chosen like a reference point because the opposite point from the local maximum is inaccessible. Furthermore, a second problem arises concerning the substrate roughness. In fact a non-homogeneous roughness will introduce an error as described in figure 3 and the real height of the NP will be difficulty measurable. As a result the substrate roughness is a determining parameter, which can degrade the measurement quality. As a consequence, in the next part a sampling method is proposed to obtain a population of isolated nanoparticles on a substrate with a minimal roughness and a sufficient NP coverage to facilitate the analysis.

\subsection{Sampling method}

Among several substrates, mica has been selected for its very low roughness $\left(R_{\mathrm{q}}=0.05 \mathrm{~nm}\right)$ and for its hydrophilic nature. A deposition method has been developed by using a spin coater and consists of two steps: a spread step at low rotation speed and a dry step at high rotation speed. The influence of the spread speed has been studied by varying this latter from $100 \mathrm{rpm}$ to $1000 \mathrm{rpm}$. Three areas with different agglomeration state are observed: the central area and the sample edges where, for all speeds, NPs agglomeration is important, and an intermediate area where, for lower speeds, samples show numerous isolated NPs. 


\subsection{Errors and uncertainty sources}

A partial uncertainty budget is proposed in table 1 and the some contributors are described in the following parts. The rest of the error sources is currently investigated.

\subsubsection{Calibration and standard homogeneity}

A surface topography standard STS2-440P from VLSI has been used to calibrate the AFM. The calibration certificate indicates a pitch oh $1803 \pm 23 \mathrm{~nm}$ and a height of $41.3 \pm 0.7 \mathrm{~nm}$. After measuring, calibration parameters are obtained through an image treatment software (SPIP) and inserted in the AFM control interface in order to correct instrument errors. The calibration has been regularly controlled over three measuring days and a first reproducibility test has been performed on different pattern of the standard. The peak-to-peak deviation for $\mathrm{Z}$ measurements on different position of the standard reaches $0.44 \mathrm{~nm}$. This large deviation is due to standard inhomogeneity. Indeed a second repeatability test carried out on the same patterns of the grating showed a lower deviation of $0.08 \mathrm{~nm}$ in thermal stability conditions.

\subsubsection{Scanning speed}

The scanning speed is an important parameter of the AFM imaging. The influence of this parameter on a NP height measurement has been evaluated by varying the speed on the same NP. Between 1 and $6 \mu \mathrm{m} / \mathrm{s}$, the NP is rightly imaged and about $1 \%$ discrepancy is measured on the NP height within this scan speed range. But above $4 \mu \mathrm{m} / \mathrm{s}$, risks of moving nanoparticles by the tip can occur. Moreover, from $6 \mu \mathrm{m} / \mathrm{s}$ to $50 \mu \mathrm{m} / \mathrm{s}$, overshooting phenomenon due to a lack of responsiveness of the feedback system dramatically increases the height measurement errors. So, with due allowance for these considerations the most suitable scanning speed has been evaluated to $4 \mu \mathrm{m} / \mathrm{s}$.

\subsubsection{Vertical drift}

In order to minimize the uncertainties and measurement errors, the vertical drift has been tested. For that purpose, a temperature sensor was installed nearest to the tip and the sample. At the beginning of the study electronics were switched off in order to make uniform the temperature of the instrument with the opened enclosure. Then the electronics was switched on and the same pattern of the VLSI standard was imaged. The temperature was recorded and all data as well as images were saved as soon as closing enclosure. The vertical drift was evaluated by measuring the maximum of acquired images during all experiment (63 hours). Finally the thermal stability was reached after 46 hours at around $32^{\circ} \mathrm{C}$ and the vertical drift could be evaluated to $6 \mu \mathrm{m} / \mathrm{min}$ on the thermal stability plateau. For a $79.05 \mathrm{~nm}$ particle, this drift impacts the height measurement is $2.10^{-}$ ${ }^{3} \mathrm{~nm}$.
Table 1: Uncertainty budget of the AFM measurement of a NP

\begin{tabular}{|c|c|c|c|}
\hline Rank & Terms & Estimation & $\begin{array}{c}\text { Contribution } \\
\text { on the NP } \\
\text { height } \\
\text { measurement }\end{array}$ \\
\hline \multicolumn{4}{|c|}{ TYPE B } \\
\hline 1 & $\begin{array}{l}\text { Calibration of the } \\
\text { VLSI standard height }\end{array}$ & $0.7 \mathrm{~nm}$ & $0.70 \mathrm{~nm}$ \\
\hline 2 & $\begin{array}{c}\text { Scanning speed } \\
\text { influence along speed } \\
\text { axe }\left(4 \mu \mathrm{m} . \mathrm{s}^{-1}\right)\end{array}$ & $0.24 \mathrm{~nm}$ & $0.24 \mathrm{~nm}$ \\
\hline 3 & $\begin{array}{c}\text { Standard } \\
\text { homogeneity along Z } \\
\text { (standard deviation) }\end{array}$ & $0.14 \mathrm{~nm}$ & $0.14 \mathrm{~nm}$ \\
\hline 7 & Roughness $(R \mathrm{q})$ & $0.05 \mathrm{~nm}$ & $0.05 \mathrm{~nm}$ \\
\hline 8 & $\begin{array}{c}\text { Instrument } \\
\text { repeatability along } \mathrm{Z}\end{array}$ & $0.04 \mathrm{~nm}$ & $0.04 \mathrm{~nm}$ \\
\hline \multirow[t]{2}{*}{9} & $\begin{array}{l}\text { Drift along } Z \text { axis at } \\
\text { stabilized temperature }\end{array}$ & $6 \mathrm{~nm} \cdot \mathrm{min}^{-1}$ & $2.10^{-3} \mathrm{~nm}$ \\
\hline & Total U (95\%): & & $0.75 \mathrm{~nm}$ \\
\hline
\end{tabular}

\section{SEM measurements}

\subsection{SEM measurement principle}

Manfred Van Ardenne and Max Knoll proposed the first realization of the Scanning Electron Microscopy in 1938.

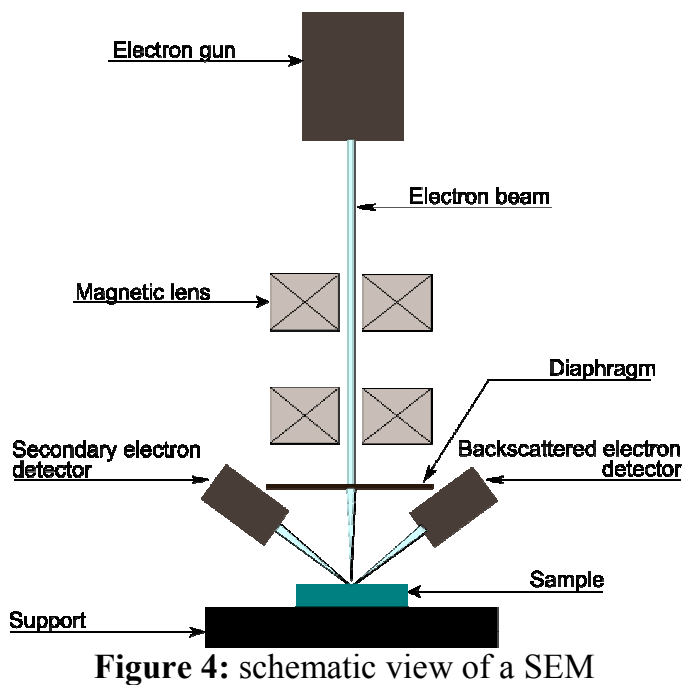

The basic principle is described in Figure 4. The electron gun produces a source of electrons emitted by a Schottky effect cathode and accelerates them to an energy in the range of 1-25 keV. Electrons are then pointed at the sample thanks to a system similar to an optical system but with using magnetic lenses and diaphragm. Interaction with the sample produces two types of electrons: 
- Secondary electrons arising from the inelastic interactions $i e$ with energy loss. They are well suitable to image the topography of the surface and the morphology of the nano-objects.

- Backscattered electrons arising from elastic interactions ie without energy loss. These electrons are primary electrons that have been scattered out of the sample by elastic collision with the nuclei of sample atoms. They are especially used for illustrating contrasts in composition of the sample and allow so a rapid discrimination of various nano-objects on the surface.

In the case of a FEG SEM, the highly focused electron beam makes it possible to obtain high resolution images. The beam diameter can be at least as small as $1 \mathrm{~nm}$ above a certain voltage. But at the end the ultimate resolution is determined by the interactions between the beam and the surface involving complex mechanisms. That is the reason why the image generated by a SEM must be carefully interpreted. Furthermore, the most commonly noted problem with a SEM is the damage caused by the beam and the exposition time. Finally the metrologist, user of SEM, has to keep in mind that the SEM is unable to give direct real measurement along $Z$ axis. The $Z$ information is just a contrast to show a $3 D$ image of the object. As a conclusion, SEM and AFM are complimentary techniques to measure a nano-object in the 3 dimensions.

\subsection{First non exhaustive list of errors and uncertainty sources}

A first list of uncertainty sources is proposed here but must be completed. Many other error sources like the dilatation of the nanoparticle under the electron beam have to be evaluated.

\subsubsection{Instrument calibration}

The SEM was calibrated with the same VLSI standard used for the AFM calibration. But in the case of the SEM calibration procedure, the pitch of $2 \mathrm{D}$ grating is involved while AFM is calibrated by means of the step height. Because no software are dedicated to this calibration procedure, the pitch grating measurement was hand-made on the two axes $\mathrm{X}$ and $\mathrm{Y}$ by using the pitch between the patterns on each line for $\mathrm{X}$-axis and each column for $\mathrm{Y}$ axis. Finally, like AFM the main error source comes from VLSI standard. The provider gives an uncertainty of 23 $\mathrm{nm}$.

\subsubsection{Leading edge distortion}

The calibration procedure by lateral measurements of the 2D grating standard showed an artefact occurring on the image edge, commonly observed and called the leading edge distortion [2]: on each line of the image, the first pixels (from the left to the right edge of the image) are smaller than the pixel given by the instrument. This default exists on each image acquired by the SEM. To be free from this default, the image must be cropped of 200 pixels (whatever the pixel size) from the left edge.

\subsubsection{Pixel size and lateral resolution limit}

These calculations are made for a 3072 × 2304 pixels image with a pixel size of $6.914 \mathrm{~nm}$, that is to say, corresponding to an image of $21.24 \times 15.93 \mu \mathrm{m}^{2}$. This image size represents a good compromise to obtain the best measurement on the largest number of nanoparticles. But anyway the pixel size will impact directly the measurement of the lateral diameter. The lateral resolution limit $S$ is equal to 1.5 times the pixel size: $10.37 \mathrm{~nm}$.

\section{Images treatment}

The same population of $\mathrm{SiO}_{2}$ nanoparticles synthesized by Stöber method [3] was deposited by the spin coating method described above and imaged by both techniques, AFM and SEM. The image processing was performed in two steps by means of the Mountain's Map (Digital Surf) software and a homemade program (Nanoselect) developed on Matlab (MathWorks) enabling a semiautomatic selection of nanoparticles to be counted in the histogram. In this manner, all artefacts and agglomerates are eliminated. This program was adapted to both microscopy techniques.

\subsection{Mountain's Map (Digital Surf) pre-treatment}

Both AFM and SEM images give topographical data about the sample. A sub-nanometer resolution along Zaxis can be reached with AFM whereas the SEM supplies no metrological information on the height but provides an accurate lateral measurement thanks to the collection of secondary electrons. So, images from SEM have to be treated differently from AFM images.

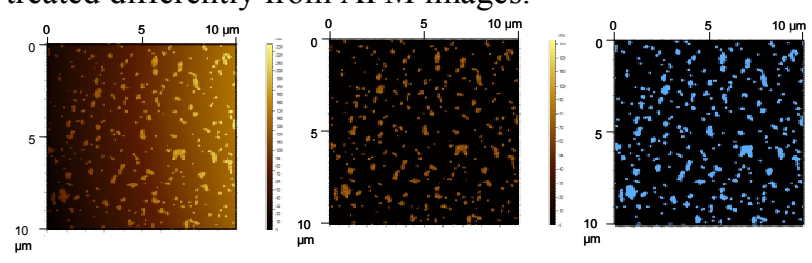

Figure 5: from the left to the right, raw, levelled and binarized AFM images of $\mathrm{SiO}_{2}$ NPs deposited by spin coating method

The AFM software (Nanoscope, Bruker) supplies three raw images (topography, amplitude and phase) after the acquisition. The topography image is so not levelled, and the first step of the treatment corrects this point through the line wise correction function from Mountain's Map software. Then, this later is saved and binarized (Figure 5) thanks to Mountain's Map's binarization function with the aim to separate the element to be analyzed (nanoparticles) from the sample surface (substrate). The phase image is saved too.

SEM images have not to be levelled because only lateral measurements correspond to our data of interest. So, in this case, only the binarization step is carried out. Nevertheless this step can be an important source of errors for SEM images. In fact, the binarization enables to differentiate the nanoparticles from the substrate by fitting a threshold between both. But this limit may be quite difficult to be determined due to noise. Indeed, the edge of the nanoparticles merges often with the noise. The threshold must be carefully fixed because it straight 
impacts the calculation of the NP lateral diameter in the case of SEM measurements.

\subsection{Nanoselect software developed on Matlab (Mathworks)}

\subsubsection{AFM image semi-automatic treatment and nanoparticle diameter calculations from height measurements}

As described in the paragraph 2, for AFM images of NPs, diameter measurements and distribution are calculated by taking into account the maximum height of each isolated nanoparticle. For this calculation of height, the reference plane is determined from the binary image, which is used as a mask on the topography image. Then the mean surface height is deduced from the roughness calculation (Figure 3).

But this binary mask is also used to localize and calculate some parameters linked to nanoparticles like the centroid or the bounding box, and the perimeter or area via the regionprops function. These data are saved in an array.

The height measurement is obviously not available on the binary image, and this measurement on each particle is obtained from the topography image where the mean surface height is put to zero: each element position is well known thanks to the previous step, and the height measurement corresponds to the maximum height point of the NP. This latter data is saved for each element in the array.

Then, each element of the image, single nanoparticles as well as aggregates and artefacts are shown (figure 6) to user through a Graphical User Interface (GUI). This step allows the user to choose objects, which have to be taken into account in the final histogram. Only single nanoparticles and their parameters are accepted.

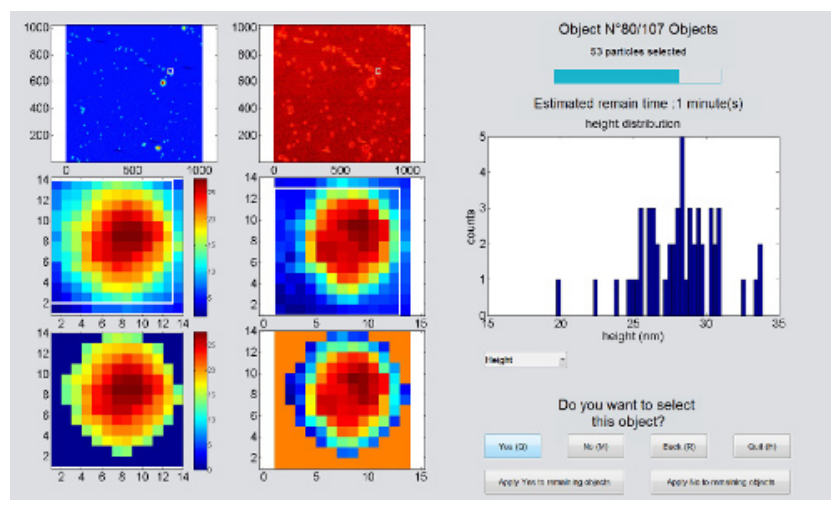

Figure 6: Screen copy of the GUI developed under Matlab showing the whole image (left topography, and right, phase) and the particle and surrounding zoom. On the right part of the interface, the histogram under construction, the number of NPs and the selection pushbuttons are shown.

Finally the final size distribution histogram is shown to the user, with the mean value and the standard deviation. The user has the choice to fit the histogram with a normal or a lognormal distribution [4].

In our study, four AFM images of $\mathrm{SiO}_{2}$ have been studied in order to obtain a sufficient number of single NPs. The size distribution is reported in Figure 7.

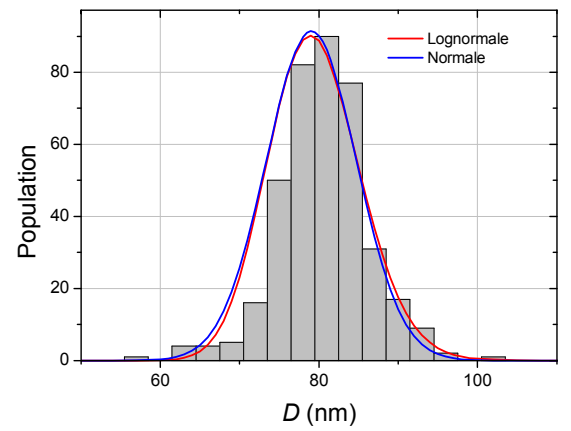

Figure 7: Histogram of height measurements by AFM of the $\mathrm{SiO}_{2}$ spherical NP population with the normal and log-normal laws fitted from the mean value and the standard deviation. Both normal and lognormal laws cannot be clearly differentiated.

The mean diameter value obtained from counting 389 NPs is $D_{\mathrm{m} A F M}=79.05 \mathrm{~nm}$ and the standard deviation of the size distribution is $\sigma_{A F M}=5.67 \mathrm{~nm}$.

\subsubsection{SEM image semi-automatic treatment and nanoparticle diameter calculations from area measurements}

As described in paragraph 4.1, the objective is to measure the lateral dimension of NPs from SEM images. The binarized image is used as a mask and in the same way than the AFM images. As previously mentioned, many noise pixels can occur on the image. So, a filter is used to eliminate these pixels. In many cases, the size of noisy areas is roughly 5 pixels and that is very small compared to nanoparticle size ( 100 pixels for a $3072 \times 2304$ pixel image and a pixel size of $6.914 \mathrm{~nm}$ ).

The second mask, without noise pixels, is then used to localize nanoparticles. In the same manner than AFM images, the regionprops function gives positions and parameters available from the binary image like the area, the perimeter, or the equivalent diameter of each nanoobject. This latter parameter is described as equivalent because it represents the diameter of a disk with a surface similar to the binarized object. However, in our study, as the particles are assumed to be spherical, the projected area is also a disk, and the equivalent diameter is similar to the diameter of the particle.

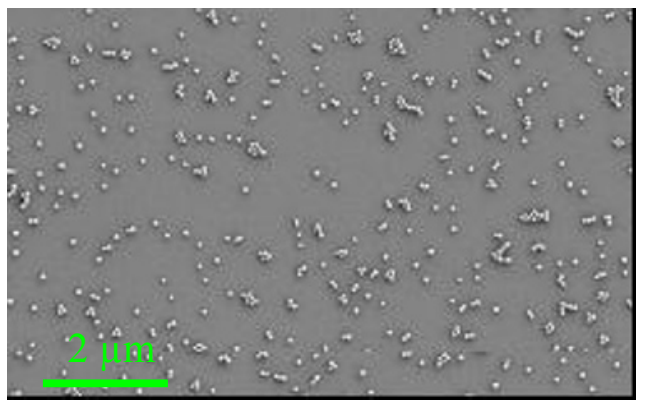

Figure 8: $\mathrm{SEM}$ image of $\mathrm{SiO}_{2}$ nanoparticles deposited by spin coating method described in the paragraph 2.1.

Only one SEM image, shown in Figure 8, was analysed and made it possible to obtain a sufficient number of nanoparticles. 


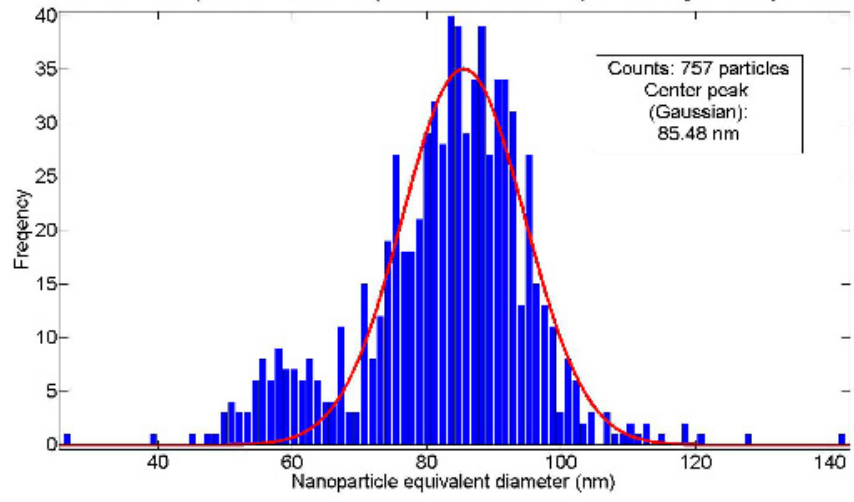

Figure 9: Histogram of diameter measurements by SEM of the $\mathrm{SiO}_{2}$ spherical-shaped NP population, fitted by a normal law.

The histogram of size distribution is reported in Figure 9. 757 single nanoparticles was counted. The mean diameter value is $D_{\mathrm{mSEM}}=85.48 \mathrm{~nm}$ and the standard deviation of the size distribution is $\sigma_{\mathrm{SEM}}=9.09 \mathrm{~nm}$. A second mode seems to occur around 59 $\mathrm{nm}$. Some ongoing measurements will enable to understand why this mode is non detectable by AFM or if it's a matter of artefacts.

\subsubsection{AFM and SEM nanoparticle diameter measurements comparison}

Two distributions have been obtained for both microscopy techniques on the same sample, and so on the same nanoparticle population. AFM gives a result of $79.05 \mathrm{~nm}$ (size distribution is $5.67 \mathrm{~nm}$ ) and MEB gives a result of $85.48 \mathrm{~nm}$ (size distribution is $9.09 \mathrm{~nm}$ ). The uncertainty budget gives an uncertainty of $0.75 \mathrm{~nm}$ on the height measurement by AFM, and a partial uncertainty of $25.23 \mathrm{~nm}$ could be given for SEM lateral diameter measurements. This latter has been calculated on an image of $3072 \times 2304$ pixels corresponding to a 25.59 $\mathrm{x} 15.93 \mu \mathrm{m}^{2}$ surface. Although the uncertainty linked to SEM measurement is large, results performed with both microscopy techniques are consistent.

\section{Conclusions and perspective}

First comparisons between platform CARMEN's AFM and SEM measurements on the same NPs population give consistent measurements, despite of a large uncertainty given on SEM measurements. In the near future, LNE's metrological AFM will make it possible to give a better uncertainty on height measurements of the standard as well as much better uncertainty on the pitch. That will noticeably reduce AFM vertical measurement uncertainty and the lateral measurement uncertainty carried out with SEM.

In order to well understand the discrepancies observed with both techniques, measurements on exactly the same set of nanoparticles are required. This study is in progress at LNE.

\section{References}

[1] Binnig G, Quate C F, Gerber C, "Atomic force microscopy", 1986, Phys. Rev. Lett. 56 930-934.

[2] Klein T., Buhr E., Johnsen K-P., Frase C.G.,"Traceable measurement of nanoparticle size using a scanning electron microscope in transmission mode (TSEM)", 2011, Meas. Sci. Technol., 2294002.

[3] Huang Y., Pemberton J.E., «Synthesis of uniform, spherical sub- $100 \mathrm{~nm}$ silica particles using a conceptual modification of the classic LaMer model», 2010, Colloids and Surfaces A: Physicochem. Eng. Aspects, 360 175-183.

[4] Nam Woong Song and al., "Uncertianty estimation of NP size distribution from a finite number data obtained by microscopic analysis", 2009, Metrologia, 46 480-488. 